Relations industrielles

Industrial Relations

\title{
Lexique du calcul économique et de l'économétrie, par André Olmi et Fortuné July, Paris, Entreprise Moderne d'Édition, 1970, 183 pages.
}

\section{Claude Autin}

Volume 26, numéro 2, 1971

URI : https://id.erudit.org/iderudit/028231ar

DOI : https://doi.org/10.7202/028231ar

Aller au sommaire du numéro

Éditeur(s)

Département des relations industrielles de l'Université Laval

ISSN

0034-379X (imprimé)

1703-8138 (numérique)

Découvrir la revue

Citer ce compte rendu

Autin, C. (1971). Compte rendu de [Lexique du calcul économique et de l'économétrie, par André Olmi et Fortuné July, Paris, Entreprise Moderne d’Édition, 1970, 183 pages.] Relations industrielles / Industrial Relations, 26(2), 512-513. https://doi.org/10.7202/028231ar

Tous droits réservés @ C Département des relations industrielles de l'Université Laval, 1971
Ce document est protégé par la loi sur le droit d'auteur. L'utilisation des services d’Érudit (y compris la reproduction) est assujettie à sa politique d'utilisation que vous pouvez consulter en ligne.

https://apropos.erudit.org/fr/usagers/politique-dutilisation/ 
sente un lexique des principaux termes ou expressions propres à cette discipline très spécialisée.

Mais c'est le chapitre VII qui traite de la législation en cette matière qui est le plus intéressant à parcourir. L'Auteur, après avoir résumé les principales lois relatives à la négociation collective dans les services publics tant au fédéral que dans les Etats où il en existe, donne un compte rendu assez détaillé des rapports des commissions d'enquête qui ont été chargées de se pencher sur ces problèmes au cours des dernières années. De telles enquêtes ont eu lieu notamment dans les Etats du Connecticut, du Michigan, de l'Illinois, de New York, du NewJersey, de la Pennsylvanie et du Maryland.

Quelques recommandations de ces commissions d'enquête retiennent principalement l'attention. Par exemple, elles se sont montrées quasi unanimes à rejeter le droit de grève pour les employés des services publics. Ainsi, celle qui a étudié la question au Michigan s'exprime ainsi : «Nous n'en sommes pas rendus au point où il faille en arriver à cette « conclusion ultime» (la grève). Nous pensons que, dans l'état actuel des choses, l'attention doit se concentrer sur l'établissement de mécanismes efficaces de négociations et de règlement des griefs sans recours à l'arbitrage obligatoire tout en continuant le maintien d'une politique d'opposition à la grève ». Cette expression d'opinion représente en quelque sorte une espèce de consensus général de la part de ces groupes d'études. Tous s'accordent à dire qu'il y a une différence marquée entre les négociations dans le secteur public et le secteur privé, même si, fondamentalement, les travailleurs recherchent des buts identiques.

Par ailleurs, il faut souligner que toutes les commissions attachent une grande importance aux commissions d'enquête (fact-finding boards) pour l'étude des conflits d'intérêts. Les recommandations de tels organismes seraient de nature à exercer une pression véritable sur les parties elles-mêmes et sur l'opinion publique.

D'ailleurs, le recours à ces commissions semble généralement donner de bons résultats. On cite le cas de l'Etat du Michigan où, en 1967, les parties ont réussi à s'entendre dans la plupart des différends sans que les commissions n'aient eu besoin de formuler des recommandations.

On est arrivé à des résultats à peu près identiques au Connecticut et dans le Massachusetts. La raison en paraît assez simple : c'est que ces enquêtes, conduites par les parties elles-mêmes avec le concours de spécialistes, ont l'avantage de poser objectivement les problèmes devant les parties, et si nécessité il y a, devant l'opinion publique, avant que les conflits se soient envenimés au point de dégénérer en surenchère ou en guerre plus ou moins totale. C'est là, selon toute apparence, une approche de problèmes majeurs dans le domaine des relations humaines dont on peut dire qu'elle est sérieuse et raisonnable.

Le secteur public tend à prendre de plus en plus d'ampleur dans la vie économique des pays fortement industrialisés et surtout urbanisés. On estime que, aux Etats-Unis, il y a aujourd'hui un travailleur sur six qui y est employé. La situation n'est pas tellement différente au Canada et au Québec, et les problèmes sont de même nature.

D'autre part, sans qu'il soit nécessaire d'imiter aveuglément ce qui se fait outrefrontière dans ce domaine, il n'en reste pas moins qu'il s'agit là d'une expérience extrêmement riche et diversifiée qu'on ne peut ignorer.

L'ouvrage d'Harold Roberts, qui est pour ainsi dire une espèce de procès verbal de ce qui s'est accompli dans le domaine des relations de travail aux Etats-Unis en tant que les services publics sont concernés, mérite-t-il d'être connu et consulté? En plus de servir à l'occasion d'instrument de travail, il peut permettre de faire d'intéressantes comparaisons et de provoquer une réflexion profitable chez tous ceux qui sont engagés dans la tâche difficile et hasardeuse d'orienter et de guider les employeurs et les travailleurs des services publics.

André ROY

\section{Lexique du calcul économique et de l'économétrie, par André OLMI et Fortuné JULY, Paris, Entreprise Mo- derne d'Edition, 1970, 183 pages.}

Ce dictionnaire miniature de 400 définitions concernant le calcul économique et l'économétrie veut être à la fois «un moyen de révision pour l'étudiant, un 
instrument d'initiation, un aide-mémoire méthodologique pour le praticien 》. Au prix d'un peu plus de 5 dollars, ce livre vaut-il la peine d'être acheté? Tout dépend de a fortune et de la formation de l'acheteur éventuel que nous éclairerons en ajoutant ce qui suit.

Un juste équilibre entre les mathématiques, la statistique et l'économétrie proprement dite est atteint. Les définitions et les exemples sont-ils suffisants pour que le non-spécialiste saisisse l'essentiel? Je ne puis me prononcer avec certitude tant la plupart des termes définis sont déjà chargés de sens et d'expérience pour moi. Il me semble, malgré tout, que les mots et les exemples restent assez simples pour être compris d'un public instruit. Les auteurs ont voulu introduire à la fois les idées fondamentales et les nouveautés techniques et je crois qu'ils ont réussi mais on comprend mal l'absence de définition pour la variance d'une variable aléatoire et pour le modèle de régression.

La présentation rend la lecture facile et les deux index facilitent la recherche. Nous n'avons noté qu'une erreur de notation; dans la définition de l'inégalité de Bienaymé-Tchebycheff (p. 97), il aurait fallu écrire \& entre... X et sa valeur moyenne $\mu \gg$. Notons aussi que M. Léontief écrit son nom avec un seul $f$ (p. 162) et que la «production intérieure nette » en général, est la production intérieure brute moins les amortissements économiques du capital $»$ et non pas «la production moins les investissements ». Enfin, nous trouvons que l'affirmation suivante est un peu forte: «Elle (la recherche opérationnelle) concerne l'ensemble des recherches fondamentales et appliquées dans le domaine de l'économie », (p. 151). Quel impérialisme!

En bref, il s'agit d'un lexique utile pour son prix. Il sera le bienvenu dans les bibliothèques canadiennes où il nous aidera surtout à franciser notre vocabulaire technique.

\section{Claude AUTIN}

Industrial and Organizational Psychology, by Abraham K. Korman, New Jersey, Prentice Hall Inc., Englewood Cliffs, $1971,398 \mathrm{pp}$.

Lorsqu'on ouvre un volume en psychologie industrielle, on s'attend à ce qu'on y traite de sélection et de formation du personnel en vue d'accroître le rendement et de faciliter une meilleure adaptation de l'homme au travail. Cette attente s'inscrit nettement dans l'optique traditionnelle de l'application de la psychologie aux solutions des problèmes humains du travail.

Sans délaisser cette orientation, l'auteur de cet ouvrage élargit considérablement le champ de cette discipline en essayant d'englober l'ensemble des facteurs individuels et des variables organisationnelles qui peuvent rendre compte d'un rendement élevé ou d'un haut degré de satisfaction au travail. Il ne cherche pas à se cantonner dans la dimension pratique de l'application de la discipline en contexte organisationnel.

Dans les premiers chapitres, il discute des méthodes et des problèmes inhérents à la recherche en psychologie du travail. Comme toute recherche sérieuse suppose un cadre de référence théorique adéquat, l'auteur s'est imposé la tâche de retracer et critiquer d'une façon constructive l'arrière-plan théorique qui a présidé à l'étude de chaque sujet qui compose les différents chapitres de son ouvrage. C'est sous cet éclairage théorique revisé qu'il reprend les principaux thèmes tels que la motivation, la satisfaction et le comportement général de l'individu au sein des organisations.

Une revue critique de la théorie entraine l'auteur dans une seconde revision ; cette fois, celle des techniques de mesures qu'on utilise habituellement au cours de la sélection et de la notation du personnel, de l'appréciation du rendement ou des résultats de la formation, etc...

Cette révision tant au plan de la théorie qu'à celui des méthodes s'accompagne d'une présentation des travaux récents dont la plupart des ouvrages antérieurs ne pouvaient faire état et qui ont été publiés au cours de la dernière décennie dans les revues et périodiques spécialisés en psychologie industrielle et en sciences du comportement.

Pour les lecteurs de cette revue et pour tous ceux qui s'intéressent à la direction du personnel et qui peuvent difficilement se procurer ces périodiques, la lecture de cet ouvrage serait une occasion d'enrichir leurs connaissances et possiblement d'innover dans la pratique quotidienne des tâches de direction de personnel.

Laurent BELANGER 